\title{
The Study of Wave Propagation in a Shell with Soft Nonlinearity and with a Viscous Liquid Inside
}

\author{
L. I. Mogilevich, S. V. Ivanov
}

This article is devoted to studying longitudinal deformation waves in physically nonlinear elastic shells with a viscous incompressible fluid inside them. The impact of construction damping on deformation waves in longitudinal and normal directions in a shell, and in the presence of surrounding medium are considered.

The presence of a viscous incompressible fluid inside the shell and the impact of fluid movement inertia on the wave velocity and amplitude are taken into consideration. In the case of a shell filled with a viscous incompressible fluid, it is impossible to study deformation wave models by qualitative analysis methods. This makes it necessary to apply numerical methods. The numerical study of the constructed model is carried out by means of a difference scheme analogous to the Crank-Nickolson scheme for the heat conduction equation. The amplitude and velocity do not change in the absence of surrounding medium impact, construction damping in longitudinal and normal directions, as well as in the absence of fluid impact. The movement occurs in the negative direction, which means that the movement velocity is subsonic. The numerical experiment results coincide with the exact solution, therefore, the difference scheme and the modified Korteweg-de Vries - Burgers equation are adequate.

Keywords: nonlinear waves, elastic cylinder shell, viscous incompressible fluid, Crank-Nickolson difference scheme

Received April 10, 2019

Accepted July 15, 2019

The work was supported by the RFBR grant No. 19-01-00014a.

Lev I. Mogilevich

mogilevichli@gmail.com

Yuri Gagarin State Technical University of Saratov

ul. Politechnicheskaya 77, Saratov, 410054 Russia

Sergey V. Ivanov

evilgraywolf@gmail.com

Saratov State University

ul. Astrakhanskaya 83, Saratov, 410012 Russia

RUSSIAN JOURNAL OF NONLINEAR DYNAMICS, 2019, 15(3), 233-250 


\section{Introduction}

The problem of wave propagation in gas dynamics and elastic shells theory is studied using linearized equations. The disturbance propagation velocity is taken to be constant and equal to the sound propagation velocity in an undisturbed medium. However, a number of cases in spite of small values of dependent variables are defined by the dependence of the disturbance propagation velocity on dependent variables values and are studied on the basis of nonlinear equations. These investigations are carried out by perturbation methods.

The study of the wave process in elastic shells is widely applied in various technical fields. The propagation of deformation waves in elastic, viscoelastic, and nonlinear viscoelastic shells and plates was considered in [7-11]. In those papers, the case of interaction of shells and a viscous incompressible fluid is not considered. References [12-14] consider the case of interaction of shells and a viscous incompressible fluid without taking into account wave phenomena; neither were local terms of inertia influence investigated. Various methods are used to solve related and unrelated problems.

When solving unrelated problems, the motion of a fluid interacting with a rigid body is considered. The stress exerted by the fluid on the rigid body, friction and pressure are determined. Thus, it is assumed that there is no influence of the deformation of the shell on the movement of the fluid [15-19]. The parameters obtained are substituted into the equations of the dynamics of an elastic body, then longitudinal and normal (deflection) displacements are found. Thus, the stress-strain state of the elastic structure, as the aim of the uncoupled problem, is determined.

In the case of a related problem, the equations of the dynamics of an elastic body and a fluid are solved simultaneously, taking into account the boundary conditions on impermeable surfaces. This approach has been applied to the study of hydroelastic vibrations [20], as well as in this article to the study of nonlinear deformation waves of elastic shells containing a viscous incompressible fluid, taking into account the inertia of its motion.

It is impossible to investigate models of deformation waves using methods of qualitative analysis in the case of a shell filled with a viscous incompressible fluid [1]. This makes it necessary to apply numerical methods.

This article examines the influence of structural damping in the longitudinal and normal direction, surrounding elastic medium, viscous incompressible fluid inside the shell as well as the inertia of fluid motion on the wave amplitude and velocity.

In the course of investigation the numerical study of the constructed model was carried out by using a difference scheme for an equation similar to the Crank-Nicholson scheme for the heat equation [21].

\section{Defining and resolving relations of the physically nonlinear theory of shells}

Let us consider an axisymmetric cylindrical shell. Denote: $h_{0}$ is the thickness of the shell; $R$ is the radius of the medium surface; $R_{1}$ is the radius of the inner surface; $U$ is the longitudinal elastic displacement; $W$ is deflection directed to the center of curvature.

Longitudinal deformation waves in an infinitely long shell are featured in the long-wave low-frequency model described by the Kirchhoff-Love theory.

A. A. Ilyushin's deformation theory of plasticity $[2,22]$ connects the components of the stress tensor $\sigma_{x}, \sigma_{\Theta}$ with the components of the strain tensor $\varepsilon_{x}, \varepsilon_{\Theta}$, and the square of the 
strain intensity $\varepsilon_{u}[3,23]$

$$
\begin{gathered}
\sigma_{x}=\frac{E}{1-\mu_{0}^{2}}\left(\varepsilon_{x}+\mu_{0} \varepsilon_{\Theta}\right)\left(1-\frac{m}{E} \varepsilon_{u}^{2}\right) ; \quad \sigma_{\Theta}=\frac{E}{1-\mu_{0}^{2}}\left(\varepsilon_{\Theta}+\mu_{0} \varepsilon_{x}\right)\left(1-\frac{m}{E} \varepsilon_{u}^{2}\right) ; \\
\varepsilon_{u}^{2}=\frac{4}{3}\left(\mu_{1}\left(\varepsilon_{x}^{2}+\varepsilon_{\Theta}^{2}\right)-\mu_{2} \varepsilon_{x} \varepsilon_{\Theta}\right) ; \\
\mu_{1}=\frac{1}{3}\left[1+\frac{\mu_{0}}{\left(1-\mu_{0}\right)^{2}}\right] ; \quad \mu_{2}=\frac{1}{3}\left[1-\frac{2 \mu_{0}}{\left(1-\mu_{0}\right)^{2}}\right]
\end{gathered}
$$

where $E$ is Young's modulus, $m$ is a material constant determined from tensile or compression experiments, and $\mu_{0}$ is Poisson's ratio of shell material.

We write down the connection between deformations and elastic displacements in the form of [4]

$$
\varepsilon_{x}=\frac{\partial U}{\partial x}+\frac{1}{2}\left(\frac{\partial W}{\partial x}\right)^{2}-z \frac{\partial^{2} W}{\partial x^{2}} ; \quad \varepsilon_{\Theta}=-\frac{W}{R},
$$

where $x$ is the longitudinal coordinate along the median surface and $z$ is the normal coordinate in the shell $\left(-\frac{h_{0}}{2} \leqslant z \leqslant \frac{h_{0}}{2}\right)$.

The square of the intensity of deformations is

or

$$
\begin{aligned}
\varepsilon_{u}^{2} & =\frac{4}{3}\left\langle\mu_{1}\left\{\left[\frac{\partial U}{\partial x}+\frac{1}{2}\left(\frac{\partial W}{\partial x}\right)^{2}-z \frac{\partial^{2} W}{\partial x^{2}}\right]^{2}+\frac{W^{2}}{R^{2}}\right\}+\right. \\
& \left.+\mu_{2} \frac{W}{R}\left[\frac{\partial U}{\partial x}+\frac{1}{2}\left(\frac{\partial W}{\partial x}\right)^{2}-z \frac{\partial^{2} W}{\partial x^{2}}\right]\right\rangle
\end{aligned}
$$

$$
\begin{aligned}
\varepsilon_{u}^{2} & =\frac{4}{3}\left\langle\mu_{1}\left\{\left[\frac{\partial U}{\partial x}+\frac{1}{2}\left(\frac{\partial W}{\partial x}\right)^{2}\right]^{2}+\left(\frac{W}{R}\right)^{2}\right\}+\mu_{2} \frac{W}{R}\left[\frac{\partial U}{\partial x}+\frac{1}{2}\left(\frac{\partial W}{\partial x}\right)^{2}\right]-\right. \\
& \left.-z\left[2 \mu_{1}\left(\frac{\partial U}{\partial x}+\frac{1}{2}\left(\frac{\partial W}{\partial x}\right)^{2}\right)+\mu_{2} \frac{W}{R}\right] \frac{\partial^{2} W}{\partial x^{2}}+z^{2} \mu_{1}\left(\frac{\partial^{2} W}{\partial x^{2}}\right)^{2}\right\rangle .
\end{aligned}
$$

Let us define the forces in the middle surface of the shell and the moment according to the following formulas:

where

$$
N_{x}=\int_{-\frac{h_{0}}{2}}^{\frac{h_{0}}{2}} \sigma_{x} d z ; \quad N_{\Theta}=\int_{-\frac{h_{0}}{2}}^{\frac{h_{0}}{2}} \sigma_{\Theta} d z ; \quad M_{x}=\int_{-\frac{h_{0}}{2}}^{\frac{h_{0}}{2}} \sigma_{x} z d z
$$

$$
\begin{aligned}
\int_{-\frac{h_{0}}{2}}^{\frac{h_{0}}{2}}\left[1-\frac{m}{E} \varepsilon_{u}^{2}\right] d z & =h_{0}\left\langle 1-\frac{m}{E} \frac{4}{3}\left\{\mu_{1}\left[\left(\frac{\partial U}{\partial x}+\frac{1}{2}\left(\frac{\partial W}{\partial x}\right)^{2}\right)^{2}+\left(\frac{W}{R}\right)^{2}\right]+\right.\right. \\
& \left.\left.+\mu_{2} \frac{W}{R}\left[\frac{\partial U}{\partial x}+\frac{1}{2}\left(\frac{\partial W}{\partial x}\right)^{2}\right]+\frac{h_{0}^{2}}{12} \mu_{1}\left(\frac{\partial^{2} W}{\partial x^{2}}\right)^{2}\right\}\right\rangle \\
\int_{-\frac{h_{0}}{2}}^{\frac{h_{0}}{2}} z\left[1-\frac{m}{E} \varepsilon_{u}^{2}\right] d z & =-\frac{m}{E} \frac{4}{3}\left\{-\frac{h_{0}^{3}}{12}\left[2 \mu_{1}\left(\frac{\partial U}{\partial x}+\frac{1}{2}\left(\frac{\partial W}{\partial x}\right)^{2}\right)+\mu_{2} \frac{W}{R}\right] \frac{\partial^{2} W}{\partial x^{2}}\right\}
\end{aligned}
$$




$$
\begin{aligned}
\int_{-\frac{h_{0}}{2}}^{\frac{h_{0}}{2}} z^{2}\left[1-\frac{m}{E} \varepsilon_{u}^{2}\right] d z & =\frac{h_{0}^{3}}{12}\left\langle 1-\frac{m}{E} \frac{4}{3}\left\{\mu_{1}\left[\left(\frac{\partial U}{\partial x}+\frac{1}{2}\left(\frac{\partial W}{\partial x}\right)^{2}\right)^{2}+\left(\frac{W}{R}\right)^{2}\right]+\right.\right. \\
& \left.\left.+\mu_{2} \frac{W}{R}\left[\frac{\partial U}{\partial x}+\frac{1}{2}\left(\frac{\partial W}{\partial x}\right)^{2}\right]+3 \frac{h_{0}^{2}}{20} \mu_{1}\left(\frac{\partial^{2} W}{\partial x^{2}}\right)^{2}\right\}\right\rangle
\end{aligned}
$$

Substituting (2.6) into (2.5), we find

$$
\begin{aligned}
N_{x}= & \frac{E h_{0}}{1-\mu_{0}^{2}}\left\langle\frac{\partial U}{\partial x}+\frac{1}{2}\left(\frac{\partial W}{\partial x}\right)^{2}-\mu_{0} \frac{W}{R}-\frac{m}{E} \frac{4}{3}\left\{\left[\frac{\partial U}{\partial x}+\frac{1}{2}\left(\frac{\partial W}{\partial x}\right)^{2}-\mu_{0} \frac{W}{R}\right] \times\right.\right. \\
& \times\left[\mu_{1}\left[\left(\frac{\partial U}{\partial x}+\frac{1}{2}\left(\frac{\partial W}{\partial x}\right)^{2}\right)^{2}+\left(\frac{W}{R}\right)^{2}\right]+\mu_{2}\left(\frac{\partial U}{\partial x}+\frac{1}{2}\left(\frac{\partial W}{\partial x}\right)^{2}\right) \frac{W}{R}\right]+ \\
& \left.\left.+\frac{h_{0}^{2}}{12}\left(\frac{\partial^{2} W}{\partial x^{2}}\right)^{2}\left[3 \mu_{1}\left(\frac{\partial U}{\partial x}+\frac{1}{2}\left(\frac{\partial W}{\partial x}\right)^{2}\right)+\left(\mu_{2}-\mu_{1} \mu_{0}\right) \frac{W}{R}\right]\right\}\right\rangle \\
N_{\Theta} & =\frac{E h_{0}}{1-\mu_{0}^{2}}\left\langle\mu_{0}\left[\frac{\partial U}{\partial x}+\frac{1}{2}\left(\frac{\partial W}{\partial x}\right)^{2}\right]-\frac{W}{R}-\frac{m}{E} \frac{4}{3}\left\{\left[\mu _ { 0 } \left(\frac{\partial U}{\partial x}+\right.\right.\right.\right. \\
& \left.\left.+\frac{1}{2}\left(\frac{\partial W}{\partial x}\right)^{2}\right)-\frac{W}{R}\right]\left[\mu_{1}\left[\left(\frac{\partial U}{\partial x}+\frac{1}{2}\left(\frac{\partial W}{\partial x}\right)^{2}\right)^{2}+\left(\frac{W}{R}\right)^{2}\right]+\right. \\
& \left.+\mu_{2}\left(\frac{\partial U}{\partial x}+\frac{1}{2}\left(\frac{\partial W}{\partial x}\right)^{2}\right) \frac{W}{R}\right]+ \\
& \left.\left.+\frac{h_{0}^{2}}{12}\left(\frac{\partial^{2} W}{\partial x^{2}}\right)^{2}\left[3 \mu \mu_{0}\left(\frac{\partial U}{\partial x}+\frac{1}{2}\left(\frac{\partial W}{\partial x}\right)^{2}\right)+\left(\mu_{1}-\mu_{2} \mu_{0}\right)^{\frac{W}{R}}\right]\right\}\right\rangle \\
& \left.\left.+2\left(\mu_{2}-\mu_{1} \mu_{0}\right)\left[\frac{\partial U}{\partial x}+\frac{1}{2}\left(\frac{\partial W}{\partial x}\right)^{2}\right] \frac{W}{R}+\left(\mu_{1}-\mu_{2} \mu_{0}\right)\left(\frac{W}{R}\right)^{2}+3 \frac{h_{0}^{2}}{20} \mu_{1}\left(\frac{\partial^{2} W}{\partial x^{2}}\right)^{2}\right\}\right\rangle . \\
M_{x}= & \frac{E h_{0}^{3}}{12\left(1-\mu_{0}^{2}\right)} \frac{\partial^{2} W}{\partial x^{2}}\left\langle 1-\frac{m}{E} \frac{4}{3}\left\{3 \mu _ { 1 } \left[\frac{\partial U}{\partial x}+\frac{1}{2}\left(\frac{\partial W}{\partial x}\right)^{2}+\right.\right.\right.
\end{aligned}
$$

Dynamic equations for shells with structural damping in the longitudinal and normal directions are written as

$$
\begin{gathered}
\frac{\partial N_{x}}{\partial x}=\rho_{0} h_{0} \frac{\partial^{2} U}{\partial t^{2}}+\varepsilon_{1} \frac{1}{l} \rho_{0} h_{0} \sqrt{\frac{E}{\rho_{0}\left(1-\mu_{0}^{2}\right)}} \frac{\partial U}{\partial t}-\left[q_{x}-W \frac{\partial q_{x}}{\partial r}+U \frac{\partial q_{x}}{\partial x}\right] \\
\frac{\partial^{2} M_{x}}{\partial x^{2}}+\frac{\partial}{\partial x}\left(\frac{\partial W}{\partial x} N_{x}\right)+\frac{1}{R} N_{\Theta}=\rho_{0} h_{0} \frac{\partial^{2} W}{\partial t^{2}}+\varepsilon_{2} \frac{l}{R^{2}} \rho_{0} h_{0} \sqrt{\frac{E}{\rho_{0}\left(1-\mu_{0}^{2}\right)}} \frac{\partial W}{\partial t}+ \\
+k_{1} \frac{h_{0}}{R^{3}} \rho_{0} h_{0} \frac{E}{\rho_{0}\left(1-\mu_{0}^{2}\right)} W-\left[q_{n}-W \frac{\partial q_{n}}{\partial r}+U \frac{\partial q_{n}}{\partial x}\right]_{R}
\end{gathered}
$$


where $t$ is time, $\varepsilon_{1}$ and $\varepsilon_{2}$ are the damping coefficients, $\rho_{0}$ is the density of shell material, $k_{1}$ is the coefficient of subgrade reaction, $q_{x}$ and $q_{n}$ are stresses of the fluid inside the circular section, $r$ and $x$ are the cylindrical coordinates, $V_{r}$ and $V_{x}$ are velocity vector projections on the axis of the coordinate system, $p$ is the pressure in the fluid, $\rho$ is the fluid density, $\nu$ is the kinematic viscosity coefficient, $\bar{n}$ is the normal to the median shell surface, and $\bar{n}_{r}, \bar{n}_{\Theta}, \bar{i}$ are the basis vectors $(r, \Theta, x)$ of the cylindrical coordinate system with its center on the geometric axis.

Substituting (2.1), (2.2), (2.5) into (2.8), we find the equations of dynamics in displacements

$$
\begin{aligned}
& \frac{E h_{0}}{1-\mu_{0}^{2}} \frac{\partial}{\partial x}\left\langle\frac{\partial U}{\partial x}+\frac{1}{2}\left(\frac{\partial W}{\partial x}\right)^{2}-\mu_{0} \frac{W}{R}-\frac{m}{E} \frac{4}{3}\left\{\left[\frac{\partial U}{\partial x}+\frac{1}{2}\left(\frac{\partial W}{\partial x}\right)^{2}-\right.\right.\right. \\
& \left.-\mu_{0} \frac{W}{R}\right]\left[\mu_{1}\left[\left(\frac{\partial U}{\partial x}+\frac{1}{2}\left(\frac{\partial W}{\partial x}\right)^{2}\right)^{2}+\left(\frac{W}{R}\right)^{2}\right]+\mu_{2}\left(\frac{\partial U}{\partial x}+\frac{1}{2}\left(\frac{\partial W}{\partial x}\right)^{2}\right) \frac{W}{R}\right]+ \\
& \left.\left.+\frac{h_{0}^{2}}{12}\left(\frac{\partial^{2} W}{\partial x^{2}}\right)^{2}\left[3 \mu_{1}\left(\frac{\partial U}{\partial x}+\frac{1}{2}\left(\frac{\partial W}{\partial x}\right)^{2}\right)+\left(\mu_{2}-\mu_{1} \mu_{0}\right) \frac{W}{R}\right]\right\}\right\rangle= \\
& =\rho_{0} h_{0} \frac{\partial^{2} U}{\partial t^{2}}+\varepsilon_{1} \frac{1}{l} \rho_{0} h_{0} \sqrt{\frac{E}{\rho_{0}\left(1-\mu_{0}^{2}\right)}} \frac{\partial U}{\partial t}-\left[q_{x}-W \frac{\partial q_{x}}{\partial r}+U \frac{\partial q_{x}}{\partial x}\right]_{R}- \\
& -\frac{E h_{0}^{3}}{12\left(1-\mu_{0}^{2}\right)} \frac{\partial^{2}}{\partial x^{2}}\left\langle\frac { \partial ^ { 2 } W } { \partial x ^ { 2 } } \left\{ 1-\frac{m}{E} \frac{4}{3}\left[3 \mu_{1}\left[\frac{\partial U}{\partial x}+\frac{1}{2}\left(\frac{\partial W}{\partial x}\right)^{2}\right]^{2}\right]+\right.\right. \\
& \left.\left.+2\left(\mu_{2}-\mu_{1} \mu_{0}\right)\left[\frac{\partial U}{\partial x}+\frac{1}{2}\left(\frac{\partial W}{\partial x}\right)^{2}\right] \frac{W}{R}+\left(\mu_{1}-\mu_{2} \mu_{0}\right)\left(\frac{W}{R}\right)^{2}+3 \frac{h_{0}^{2}}{20} \mu_{1}\left(\frac{\partial^{2} W}{\partial x^{2}}\right)^{2}\right\}\right\rangle+ \\
& +\frac{E h_{0}}{1-\mu_{0}^{2}} \frac{\partial}{\partial x}\left\langle\frac { \partial W } { \partial x } \left[\frac{\partial U}{\partial x}+\frac{1}{2}\left(\frac{\partial W}{\partial x}\right)^{2}-\mu_{0} \frac{W}{R}-\frac{m}{E} \frac{4}{3}\left\{\left[\frac{\partial U}{\partial x}+\frac{1}{2}\left(\frac{\partial W}{\partial x}\right)^{2}-\right.\right.\right.\right. \\
& \left.-\mu_{0} \frac{W}{R}\right]\left[\mu_{1}\left[\left(\frac{\partial U}{\partial x}+\frac{1}{2}\left(\frac{\partial W}{\partial x}\right)^{2}\right)^{2}+\left(\frac{W}{R}\right)^{2}\right]+\mu_{2}\left(\frac{\partial U}{\partial x}+\frac{1}{2}\left(\frac{\partial W}{\partial x}\right)^{2}\right) \frac{W}{R}\right]+ \\
& \left.\left.\left.+\frac{h_{0}^{2}}{12}\left(\frac{\partial^{2} W}{\partial x^{2}}\right)^{2}\left[3 \mu_{1}\left(\frac{\partial U}{\partial x}+\frac{1}{2}\left(\frac{\partial W}{\partial x}\right)^{2}\right)+\left(\mu_{2}-\mu_{1} \mu_{0}\right) \frac{W}{R}\right]\right\}\right]\right\rangle+ \\
& +\frac{E h_{0}}{1-\mu_{0}^{2}} \frac{1}{R}\left\langle\mu_{0}\left[\frac{\partial U}{\partial x}+\frac{1}{2}\left(\frac{\partial W}{\partial x}\right)^{2}\right]-\frac{W}{R}-\frac{m}{E} \frac{4}{3}\left\{\left[\mu_{0}\left(\frac{\partial U}{\partial x}+\frac{1}{2}\left(\frac{\partial W}{\partial x}\right)^{2}\right)-\right.\right.\right. \\
& \left.-\frac{W}{R}\right]\left[\mu_{1}\left[\left(\frac{\partial U}{\partial x}+\frac{1}{2}\left(\frac{\partial W}{\partial x}\right)^{2}\right)^{2}+\left(\frac{W}{R}\right)^{2}\right]+\mu_{2}\left(\frac{\partial U}{\partial x}+\frac{1}{2}\left(\frac{\partial W}{\partial x}\right)^{2}\right) \frac{W}{R}\right]+ \\
& \left.\left.+\frac{h_{0}^{2}}{12}\left(\frac{\partial^{2} W}{\partial x^{2}}\right)^{2}\left[3 \mu_{1} \mu_{0}\left(\frac{\partial U}{\partial x}+\frac{1}{2}\left(\frac{\partial W}{\partial x}\right)^{2}\right)+\left(\mu_{1}-\mu_{2} \mu_{0}\right) \frac{W}{R}\right]\right\}\right\rangle= \\
& =\rho_{0} h_{0} \frac{\partial^{2} W}{\partial t^{2}}+\varepsilon_{2} \frac{1}{R^{2}} \rho_{0} h_{0} \sqrt{\frac{E}{\rho_{0}\left(1-\mu_{0}^{2}\right)}} \frac{\partial W}{\partial t}+ \\
& +k_{1} \frac{h_{0}}{R^{3}} \rho_{0} h_{0} \frac{E}{\rho_{0}\left(1-\mu_{0}^{2}\right)} W-\left[q_{n}-W \frac{\partial q_{n}}{\partial r}+U \frac{\partial q_{n}}{\partial x}\right]_{R} .
\end{aligned}
$$




\section{The asymptotic method for studying the equations of shells with a fluid}

For wave problems, the shell is assumed to be infinite. For longitudinal waves in the shell, dimensionless variables and parameters are introduced. We take the wavelength $l$ for the characteristic length, and $u_{m}, w_{m}$ are the characteristic values of the elastic displacements

$$
W=w_{m} u_{3}, \quad U=u_{m} u_{1}, \quad x^{*}=\frac{x}{l}, \quad t^{*}=\frac{c_{0}}{l} t, \quad r^{*}=\frac{r}{R} .
$$

$c_{0}=\sqrt{\frac{E}{\rho\left(1-\mu_{0}\right)}}$ is the propagation velocity of longitudinal elastic waves in the shell. Set

$$
\begin{gathered}
\frac{h_{0}}{R}=\varepsilon \ll 1, \quad \frac{R^{2}}{l^{2}}=O(\varepsilon), \quad \frac{w_{m}}{h_{0}}=O(1), \quad \frac{\varepsilon_{1}}{\varepsilon}=O(1), \quad \frac{\varepsilon_{2}}{\varepsilon}=O(1), \\
\frac{u_{m}}{l} \frac{R}{h_{0}}=O(1), \quad \frac{m \varepsilon}{E}=O(1), \quad \frac{h_{0}^{2}}{l^{2}}=\frac{h_{0}^{2}}{R^{2}} \cdot \frac{R^{2}}{l^{2}}=\varepsilon^{3},
\end{gathered}
$$

where $\varepsilon$ is the small parameter of the problem. In these variables, Eqs. (2.9) take the form

$$
\begin{aligned}
& c_{0}^{2} \rho_{0} h_{0} \frac{1}{l} \frac{\partial}{\partial x^{*}}\left\langle\left(\frac{u_{m}}{l} \frac{\partial u_{1}}{\partial x^{*}}+\frac{1}{2} \frac{R^{2}}{l^{2}}\left(\frac{w_{m}}{R}\right)^{2}\left(\frac{\partial u_{3}}{\partial x^{*}}\right)^{2}\right)-\mu_{0} \frac{w_{m}}{R} u_{3}-\right. \\
& -\frac{m}{E} \frac{4}{3}\left\{[ ( \frac { u _ { m } } { l } \frac { \partial u _ { 1 } } { \partial x ^ { * } } + \frac { 1 } { 2 } \frac { R ^ { 2 } } { l ^ { 2 } } ( \frac { w _ { m } } { R } ) ^ { 2 } ( \frac { \partial u _ { 3 } } { \partial x ^ { * } } ) ^ { 2 } ) - \mu _ { 0 } \frac { w _ { m } } { R } u _ { 3 } ] \left[\mu _ { 1 } \left[\left(\frac{u_{m}}{l} \frac{\partial u_{1}}{\partial x^{*}}+\right.\right.\right.\right. \\
& \left.\left.+\frac{1}{2} \frac{R^{2}}{l^{2}}\left(\frac{w_{m}}{R}\right)^{2}\left(\frac{\partial u_{3}}{\partial x^{*}}\right)^{2}\right)^{2}+\left(\frac{w_{m}}{R}\right)^{2} u_{3}^{2}\right]+\mu_{2}\left(\frac{u_{m}}{l} \frac{\partial u_{1}}{\partial x^{*}}+\right. \\
& \left.\left.+\frac{1}{2} \frac{R^{2}}{l^{2}}\left(\frac{w_{m}}{R}\right)^{2}\left(\frac{\partial u_{3}}{\partial x^{*}}\right)^{2}\right) \frac{w_{m}}{R} u_{3}\right]+\frac{h_{0}^{2}}{12} \frac{R^{2}}{l^{4}} \frac{w_{m}^{2}}{R^{2}}\left(\frac{\partial^{2} u_{3}}{\partial x^{* 2}}\right)^{2}\left[3 \mu _ { 1 } \left(\frac{u_{m}}{l} \frac{\partial u_{1}}{\partial x^{*}}+\right.\right. \\
& \left.\left.\left.\left.+\frac{1}{2} \frac{R^{2}}{l^{2}}\left(\frac{w_{m}}{R}\right)^{2}\left(\frac{\partial u_{3}}{\partial x^{*}}\right)^{2}\right)+\left(\mu_{2}-\mu_{1} \mu_{0}\right) \frac{w_{m}}{R} u_{3}\right]\right\}\right\rangle= \\
& =\frac{c_{0}^{2} \rho_{0} h_{0}}{l^{2}} u_{m} \frac{\partial^{2} u_{1}}{\partial t^{* 2}}+\varepsilon_{1} \frac{c_{0}^{2} \rho_{0} h_{0}}{l^{2}} u_{m} \frac{\partial u_{1}}{\partial t^{*}}-\left[q_{x}-\frac{w_{m}}{R} u_{3} \frac{\partial q_{x}}{\partial r^{*}}+\frac{u_{m}}{l} u_{1} \frac{\partial q_{x}}{\partial x^{*}}\right] ; \\
& -c_{0}^{2} \rho_{0} \frac{h_{0}}{l} \frac{h_{0}^{2}}{12} \frac{1}{l^{2}} \frac{\partial^{2}}{\partial x^{* 2}}\left\langle\frac { R } { l } \frac { w _ { m } } { R } \frac { \partial ^ { 2 } u _ { 3 } } { \partial x ^ { * 2 } } \left\{ 1-\frac{m}{E} \frac{4}{3}\left[3 \mu_{1}\left(\frac{u_{m}}{l} \frac{\partial u_{1}}{\partial x^{*}}++\frac{1}{2} \frac{R^{2}}{l^{2}}\left(\frac{w_{m}}{R}\right)^{2}\left(\frac{\partial u_{3}}{\partial x^{*}}\right)^{2}\right)^{2}+\right.\right.\right. \\
& +2\left(\mu_{2}-\mu_{1} \mu_{0}\right)\left(\frac{u_{m}}{l} \frac{\partial u_{1}}{\partial x^{*}}+\frac{1}{2} \frac{R^{2}}{l^{2}}\left(\frac{w_{m}}{R}\right)^{2}\left(\frac{\partial u_{3}}{\partial x^{*}}\right)\right) \frac{w_{m}}{R} u_{3}+\left(\mu_{1}-\mu_{2} \mu_{0}\right)\left(\frac{w_{m}}{R}\right)^{2}+ \\
& \left.\left.\left.+3 \frac{h_{0}^{2}}{20} \frac{R^{4}}{l^{4}}\left(\frac{w_{m}}{R}\right)^{2} \mu_{1}\left(\frac{\partial^{2} u_{3}}{\partial x^{*} 2}\right)^{2}\right]\right\}\right\rangle+c_{0}^{2} \rho_{0} h_{0} \frac{1}{l} \frac{\partial}{\partial x^{*}}\left\langle\frac { R } { l } \frac { w _ { m } } { R } \frac { \partial u _ { 3 } } { \partial x ^ { * } } \left[\left(\frac{u_{m}}{l} \frac{\partial u_{1}}{\partial x^{*}}+\right.\right.\right. \\
& \left.+\frac{1}{2} \frac{R^{2}}{l^{2}}\left(\frac{w_{m}}{R}\right)^{2}\left(\frac{\partial u_{3}}{\partial x^{*}}\right)\right)-\mu_{0} \frac{w_{m}}{R} u_{3}-\frac{m}{E} \frac{4}{3}\left\{\left[\left(\frac{u_{m}}{l} \frac{\partial u_{1}}{\partial x^{*}}+\right.\right.\right. \\
& \left.\left.+\frac{1}{2} \frac{R^{2}}{l^{2}}\left(\frac{w_{m}}{R}\right)^{2}\left(\frac{\partial u_{3}}{\partial x^{*}}\right)\right)-\mu_{0} \frac{w_{m}}{R} u_{3}\right]\left[\mu _ { 1 } \left[\left(\frac{u_{m}}{l} \frac{\partial u_{1}}{\partial x^{*}}+\right.\right.\right.
\end{aligned}
$$




$$
\begin{aligned}
& \left.\left.+\frac{1}{2} \frac{R^{2}}{l^{2}}\left(\frac{w_{m}}{R}\right)^{2}\left(\frac{\partial u_{3}}{\partial x^{*}}\right)\right)^{2}+\left(\frac{w_{m}}{R}\right)^{2} u_{3}^{2}\right]+\mu_{2}\left(\frac{u_{m}}{l} \frac{\partial u_{1}}{\partial x^{*}}+\right. \\
& \left.\left.+\frac{1}{2} \frac{R^{2}}{l^{2}}\left(\frac{w_{m}}{R}\right)^{2}\left(\frac{\partial u_{3}}{\partial x^{*}}\right)\right) \frac{w_{m}}{R} u_{3}\right]+\frac{h_{0}^{2}}{12} \frac{R^{2}}{l^{4}}\left(\frac{w_{m}}{R}\right)^{2}\left(\frac{\partial^{2} u_{3}}{\partial x^{*} 2}\right)^{2}\left[3 \mu _ { 1 } \left(\frac{u_{m}}{l} \frac{\partial u_{1}}{\partial x^{*}}+\right.\right. \\
& \left.\left.\left.\left.\left.+\frac{1}{2} \frac{R^{2}}{l^{2}}\left(\frac{w_{m}}{R}\right)^{2}\left(\frac{\partial u_{3}}{\partial x^{*}}\right)\right)\left(\mu_{2}-\mu_{1} \mu_{0}\right) \frac{w_{m}}{R} u^{3}\right]\right\}\right]\right\rangle+ \\
& +c_{0}^{2} \rho_{0} h_{0} \frac{1}{R}\left\langle\mu_{0}\left(\frac{u_{m}}{l} \frac{\partial u_{1}}{\partial x^{*}}+\frac{1}{2} \frac{R^{2}}{l^{2}}\left(\frac{w_{m}}{R}\right)^{2}\left(\frac{\partial u_{3}}{\partial x^{*}}\right)^{2}\right)-\frac{w_{m}}{R} u_{3}-\right. \\
& -\frac{m}{E} \frac{4}{3}\left\{[ \mu _ { 0 } ( \frac { u _ { m } } { l } \frac { \partial u _ { 1 } } { \partial x ^ { * } } + \frac { 1 } { 2 } \frac { R ^ { 2 } } { l ^ { 2 } } ( \frac { w _ { m } } { R } ) ^ { 2 } ( \frac { \partial u _ { 3 } } { \partial x ^ { * } } ) ^ { 2 } ) - \frac { w _ { m } } { R } u _ { 3 } ] \left[\mu _ { 1 } \left[\left(\frac{u_{m}}{l} \frac{\partial u_{1}}{\partial x^{*}}+\right.\right.\right.\right. \\
& \left.\left.+\frac{1}{2} \frac{R^{2}}{l^{2}}\left(\frac{w_{m}}{R}\right)^{2}\left(\frac{\partial u_{3}}{\partial x^{*}}\right)^{2}\right)^{2}+\left(\frac{w_{m}}{R}\right)^{2} u_{3}^{2}\right]+\mu_{2}\left(\frac{u_{m}}{l} \frac{\partial u_{1}}{\partial x^{*}}+\right. \\
& \left.\left.+\frac{1}{2} \frac{R^{2}}{l^{2}}\left(\frac{w_{m}}{R}\right)^{2}\left(\frac{\partial u_{3}}{\partial x^{*}}\right)^{2}\right) \frac{w_{m}}{R} u_{3}\right]+\frac{h_{0}^{2}}{12} \frac{R^{2}}{l^{4}} \frac{w_{m}^{2}}{R^{2}}\left(\frac{\partial^{2} u_{3}}{\partial x^{* 2}}\right)^{2}\left[3 \mu _ { 1 } \mu _ { 0 } \left(\frac{u_{m}}{l} \frac{\partial u_{1}}{\partial x^{*}}+\right.\right. \\
& \left.\left.\left.\left.+\frac{1}{2} \frac{R^{2}}{l^{2}}\left(\frac{w_{m}}{R}\right)^{2}\left(\frac{\partial u_{3}}{\partial x^{*}}\right)^{2}\right)-\left(\mu_{1}-\mu_{2} \mu_{0}\right) \frac{w_{m}}{R} u_{3}\right]\right\}\right)= \\
& =\rho_{0} h_{0} \frac{c_{0}^{2}}{l^{2}} w_{m} \frac{\partial^{2} u_{3}}{\partial t^{* 2}}+\varepsilon_{2} \rho_{0} h_{0} \frac{c_{0}^{2}}{R^{2}} w_{m} \frac{\partial u_{3}}{\partial t^{*}}+k_{1} \frac{h_{0}}{R} \rho_{0} h_{0} \frac{c_{0}^{2}}{R^{2}} w_{m} u_{3}- \\
& -\left[q_{n}-\frac{w_{m}}{R} u_{3} \frac{\partial q_{n}}{\partial r^{*}}+\frac{u_{m}}{l} u_{1} \frac{\partial q_{n}}{\partial x^{*}}\right]
\end{aligned}
$$

We introduce independent variables in the form

$$
\xi=x^{*}-c t^{*}, \quad \tau=\varepsilon t^{*},
$$

where $\tau$ is fast time and $c$ is the dimensionless unknown volume. In these variables (3.4), leaving the terms of orders $\varepsilon$ and $\varepsilon^{2}$ in Eqs. (3.3) and discarding terms with higher degrees, we obtain equations [5]

$$
\begin{gathered}
\frac{\partial}{\partial \xi}\left\langle\frac{u_{m}}{l} \frac{\partial u_{1}}{\partial \xi}-\mu_{0} \frac{w_{m}}{R} u_{3}-\frac{m}{E} \frac{4}{3}\left\{( \frac { u _ { m } } { l } \frac { \partial u _ { 1 } } { \partial \xi } - \mu _ { 0 } \frac { w _ { m } } { R } u _ { 3 } ) \left[\mu _ { 1 } \left[\left(\frac{u_{m}}{l} \frac{\partial u_{1}}{\partial \xi}\right)^{2}+\right.\right.\right.\right. \\
\left.\left.\left.\left.+\left(\frac{w_{m}}{R}\right)^{2} u_{3}^{2}\right]+\mu_{2} \frac{u_{m}}{l} \frac{\partial u_{1}}{\partial \xi} \frac{w_{m}}{R} u_{3}\right]\right\}\right\rangle=\frac{u_{m}}{l}\left[c^{2} \frac{\partial^{2} u_{1}}{\partial \xi^{2}}-2 \varepsilon c \frac{\partial^{2} u_{1}}{\partial \xi \partial \tau}\right]- \\
-\frac{u_{m}}{l} \varepsilon_{1} c \frac{\partial u_{1}}{\partial \xi}-\frac{l}{\rho_{0} h_{0} c_{0}^{2}} q_{x} ; \\
\mu_{0} \frac{u_{m}}{l} \frac{\partial u_{1}}{\partial \xi}-\frac{w_{m}}{R} u_{3}-\frac{m}{E} \frac{4}{3}\left(\mu_{0} \frac{u_{m}}{l} \frac{\partial u_{1}}{\partial \xi}-\frac{w_{m}}{R} u_{3}\right)\left[\mu _ { 1 } \left[\left(\frac{u_{m}}{l} \frac{\partial u_{1}}{\partial \xi}\right)^{2}+\right.\right. \\
\left.\left.+\left(\frac{w_{m}}{R}\right)^{2} u_{3}^{2}\right]+\mu_{2} \frac{u_{m}}{l} \frac{\partial u_{1}}{\partial \xi} \frac{w_{m}}{R} u_{3}\right]=\frac{R^{2}}{l^{2}} \frac{w_{m}}{R}\left[c^{2} \frac{\partial^{2} u_{3}}{\partial \xi^{2}}-2 \varepsilon c \frac{\partial^{2} u_{3}}{\partial \xi \partial \tau}\right]- \\
-\frac{R^{2}}{l^{2}} \frac{w_{m}}{R} \varepsilon_{2} \frac{l^{2}}{R^{2}} c \frac{\partial u_{3}}{\partial \xi}+\frac{w_{m}}{R} \frac{h_{0}}{R} k_{1} u_{3}-\frac{R^{2}}{\rho_{0} h_{0} c_{0}^{2}} q_{n} .
\end{gathered}
$$


We represent the dependent variables in the form of an asymptotic expansion.

$$
u_{1}=u_{10}+\varepsilon u_{11}+\ldots, \quad u_{3}=u_{30}+\varepsilon u_{31}+\ldots
$$

We obtain a system of equations by substituting (4.1) into (3.5) and leaving the terms of order $\varepsilon$

$$
\begin{aligned}
\frac{\partial}{\partial \xi}\left\langle\frac{\partial u_{10}}{\partial \xi}-\mu_{0} \frac{w_{m} l}{u_{m} R} u_{30}\right\rangle & =c^{2} \frac{\partial^{2} u_{10}}{\partial \xi^{2}}, \\
\mu_{0} \frac{\partial u_{10}}{\partial \xi}-\frac{w_{m} l}{u_{m} R} u_{30} & =0 .
\end{aligned}
$$

From this system we get

$$
\frac{w_{m} l}{u_{m} R} u_{30}=\mu_{0} \frac{\partial u_{10}}{\partial \xi}, \quad c^{2}=1-\mu_{0}^{2} .
$$

Thus, $u_{10}$ is an arbitrary function, and the dimensionless volume $c=\left(1-\mu_{0}^{2}\right)^{1 / 2}$, therefore, the wave velocity equals $\sqrt{\frac{E}{\rho_{0}}}$, that is, the velocity of the wave in the rod. Since the shell is of infinite length,

$$
\xi=\frac{1}{l}\left(x-\sqrt{\frac{E}{\rho_{0}}} t\right) .
$$

We get a system of equations in approximation of order $\varepsilon^{2}$

$$
\begin{gathered}
\frac{\partial}{\partial \xi}\left\langle\frac{\partial u_{11}}{\partial \xi}-\mu_{0} \frac{w_{m} l}{u_{m} R} u_{31}-\frac{m}{E} \frac{4}{3}\left[\mu_{1}\left(\frac{\partial u_{10}}{\partial \xi}\right)^{3}+\right.\right. \\
+\left(\mu_{1}-\mu_{2} \mu_{0}\right) \frac{\partial u_{10}}{\partial \xi}\left(\frac{w_{m} l}{u_{m} R} u_{30}\right)^{2}+\left(\mu_{2}-\mu_{1} \mu_{0}\right) \frac{w_{m} l}{u_{m} R} u_{30}\left(\frac{\partial u_{10}}{\partial \xi}\right)^{2}- \\
\left.\left.-\mu_{1} \mu_{0}\left(\frac{w_{m} l}{u_{m} R}\right)^{3} u_{30}^{3}\right]\right\rangle=-2 c \frac{\partial^{2} u_{10}}{\partial \xi \partial \tau}+c^{2} \frac{\partial^{2} u_{11}}{\partial^{2} \xi}-\frac{\varepsilon_{1}}{\varepsilon} c \frac{\partial u_{10}}{\partial \xi}-\frac{l^{2}}{\varepsilon u_{m} \rho_{0} h_{0} c_{0}^{2}} q_{x} \\
\mu_{0} \frac{\partial u_{11}}{\partial \xi}-\frac{w_{m} l}{u_{m} R} u_{31}-\frac{m}{E} \frac{4}{3}\left(\frac{u_{m}}{l}\right)^{2}\left(\mu_{0} \frac{\partial u_{10}}{\partial \xi}+\frac{w_{m} l}{u_{m} R} u_{30}\right)\left[\mu _ { 0 } \left[\left(\frac{\partial u_{10}}{\partial \xi}\right)^{3}+\right.\right. \\
\left.\left.+\left(\frac{w_{m} l}{u_{m} R}\right)^{2} u_{30}^{2}\right]+\mu_{2} \frac{w_{m} l}{u_{m} R} \frac{\partial u_{10}}{\partial \xi} u_{30}\right]=\frac{R l}{\varepsilon} \frac{R^{2}}{l^{2}} \frac{w_{m} l}{u_{m} R} c^{2} \frac{\partial^{2} u_{30}}{\partial^{2} \xi}-\frac{\varepsilon_{2}}{\varepsilon} \frac{w_{m}}{R} \frac{l}{u_{m}} c \frac{\partial u_{30}}{\partial \xi}+k_{1} \frac{1}{\varepsilon} \frac{h_{0}}{R} \frac{w_{m} l}{u_{m} R} u_{30}-\frac{R}{\varepsilon u_{m} \rho_{0} h_{0} c_{0}^{2}} q_{n} .
\end{gathered}
$$

By substituting relation (4.3) into Eqs. (5.1), we obtain the system

$$
\begin{gathered}
\mu_{0}^{2} \frac{\partial^{2} u_{11}}{\partial^{2} \xi}-\mu_{0} \frac{w_{m} l}{u_{m} R} \frac{\partial u_{31}}{\partial \xi}= \\
=\frac{m}{E} \frac{4}{3}\left(\frac{u_{m}}{l}\right)^{2}\left(1-\mu_{0}^{2}\right)\left(\mu_{1}+\mu_{2} \mu_{0}+\mu_{1} \mu_{0}^{2}\right) 3\left(\frac{\partial u_{10}}{\partial \xi}\right)^{2} \frac{\partial^{2} u_{10}}{\partial^{2} \xi}- \\
-2 \sqrt{1-\mu_{0}^{2}} \frac{\partial^{2} u_{10}}{\partial \xi \partial \tau}-\frac{\varepsilon_{2}}{\varepsilon} \sqrt{1-\mu_{0}^{2}} \frac{\partial u_{10}}{\partial \xi}-\frac{l^{2}}{\varepsilon u_{m} \rho_{0} h_{0} c_{0}^{2}} q_{x} ;
\end{gathered}
$$




$$
\begin{aligned}
\mu_{0}^{2} \frac{\partial u_{11}}{\partial \xi}-\frac{w_{m} l}{u_{m} R} u_{31} & =\frac{1}{\varepsilon} \frac{R^{2}}{l^{2}}\left(1-\mu_{0}^{2}\right) \mu_{0} \frac{\partial^{3} u_{10}}{\partial^{3} \xi}-\frac{\varepsilon_{2}}{\varepsilon} \sqrt{1-\mu_{0}^{2}} \mu_{0} \frac{\partial^{2} u_{10}}{\partial^{2} \xi}+ \\
& +k_{1} \frac{1}{\varepsilon} \frac{h_{0}}{R} \mu_{0} \frac{\partial u_{10}}{\partial \xi}-\frac{R l}{\varepsilon u_{m} \rho_{0} h_{0} c_{0}^{2}} q_{n} .
\end{aligned}
$$

By multiplying both sides of the second equation by $\mu_{0}$ and differentiating with respect to $\xi$, we get the following equation:

$$
\begin{gathered}
\mu_{0}^{2} \frac{\partial^{2} u_{11}}{\partial^{2} \xi}-\mu_{0} \frac{w_{m} l}{u_{m} R} \frac{\partial u_{31}}{\partial \xi}=\frac{1}{\varepsilon} \frac{R^{2}}{l^{2}} \mu_{0}^{2}\left(1-\mu_{0}^{2}\right) \frac{\partial^{4} u_{10}}{\partial^{4} \xi}-\frac{\varepsilon_{2}}{\varepsilon} \sqrt{1-\mu_{0}^{2}} \mu_{0} \frac{\partial^{3} u_{10}}{\partial^{3} \xi}+ \\
+k_{1} \frac{1}{\varepsilon} \frac{h_{0}}{R} \mu_{0}^{2} \frac{\partial^{2} u_{10}}{\partial \xi^{2}}-\mu_{0} \frac{R l}{\varepsilon u_{m} \rho_{0} h_{0} c_{0}^{2}} \frac{\partial q_{n}}{\partial \xi}
\end{gathered}
$$

The left-hand side of Eqs. (3.10) and (3.11) coincide. Subtracting, term by term, the first equation of system (3.10) from Eq. (3.11), we obtain the resolving equation

$$
\begin{gathered}
2 \sqrt{1-\mu_{0}^{2}} \frac{\partial^{2} u_{10}}{\partial \xi \partial \tau}-4 \frac{m}{E \varepsilon}\left(\frac{u_{m}}{l}\right)^{2}\left(1-\mu_{0}^{2}\right)\left(\mu_{1}+\mu_{2} \mu_{0}+\mu_{1} \mu_{0}^{2}\right)\left(\frac{\partial u_{10}}{\partial \xi}\right)^{2} \frac{\partial^{2} u_{10}}{\partial^{2} \xi}+ \\
+\frac{1}{\varepsilon} \frac{R^{2}}{l^{2}} \mu_{0}^{2}\left(1-\mu_{0}^{2}\right) \frac{\partial^{4} u_{10}}{\partial^{4} \xi}-\frac{\varepsilon_{2}}{\varepsilon} \sqrt{1-\mu_{0}^{2}} \mu_{0}^{2} \frac{\partial^{3} u_{10}}{\partial^{3} \xi}+k_{1} \frac{1}{\varepsilon} \frac{h_{0}}{R} \mu_{0}^{2} \frac{\partial^{2} u_{10}}{\partial \xi^{2}}+ \\
+\frac{\varepsilon_{1}}{\varepsilon} \sqrt{1-\mu_{0}^{2}} \frac{\partial u_{10}}{\partial \xi}=-\frac{l^{2}}{\varepsilon u_{m} \rho_{0} h_{0} c_{0}^{2}}\left[q_{x}-\mu_{0} \frac{R}{l} \frac{\partial q_{n}}{\partial \xi}\right] .
\end{gathered}
$$

We divide both sides of the equation by $2 \sqrt{1-\mu_{0}^{2}}$ and get

$$
\begin{gathered}
\frac{\partial^{2} u_{10}}{\partial \xi \partial \tau}-2 \frac{m}{E \varepsilon}\left(\frac{u_{m}}{l}\right)^{2} \sqrt{1-\mu_{0}^{2}}\left(\mu_{1}+\mu_{2} \mu_{0}+\mu_{1} \mu_{0}^{2}\right)\left(\frac{\partial u_{10}}{\partial \xi}\right)^{2} \frac{\partial^{2} u_{10}}{\partial^{2} \xi}+ \\
+\frac{1}{\varepsilon} \frac{R^{2}}{l^{2}} \frac{1}{2} \mu_{0}^{2} \sqrt{1-\mu_{0}^{2}} \frac{\partial^{4} u_{10}}{\partial^{4} \xi}-\frac{\varepsilon_{2}}{\varepsilon} \frac{\mu_{0}^{2}}{2} \frac{\partial^{3} u_{10}}{\partial^{3} \xi}+k_{1} \frac{\mu_{0}^{2}}{2 \sqrt{1-\mu_{0}^{2}}} \frac{1}{\varepsilon} \frac{h_{0}}{R} \mu_{0}^{2} \frac{\partial^{2} u_{10}}{\partial \xi^{2}}+ \\
+\frac{1}{2} \frac{\varepsilon_{1}}{\varepsilon} \frac{\partial u_{10}}{\partial \xi} \frac{\mu_{0}^{2}}{2}=-\frac{1}{2 \sqrt{1-\mu_{0}^{2}}} \frac{l^{2}}{\varepsilon u_{m} \rho_{0} h_{0} c_{0}^{2}}\left[q_{x}-\mu_{0} \frac{R}{l} \frac{\partial q_{n}}{\partial \xi}\right] .
\end{gathered}
$$

The left-hand side of the resulting equation corresponds to a modified Korteweg - de Vries Burgers equation for $\frac{\partial u_{10}}{\partial \xi}$. In the absence of a fluid, the right-hand side of the equation is zero, and then a modified Korteweg-de Vries-Burgers equation is obtained. It is necessary to determine the right-hand side by solving the equations of hydrodynamics.

\section{The fluid flow stress acting on the shell surface}

The stresses of the fluid layer are defined by the formulas [6]

$$
\begin{gathered}
q_{n}=P_{r r} \cos \left(\widehat{\bar{n}, \bar{n}_{r}}\right)+P_{r x} \cos (\widehat{\bar{n}, \bar{i}}) ; \quad q_{x}=-\left[P_{r x} \cos \left(\widehat{\bar{n}, \bar{n}_{r}}\right)+P_{x x} \cos (\widehat{\bar{n}, \bar{i}})\right] ; \\
P_{r r}=-p+2 \rho \nu \frac{\partial V_{r}}{\partial r} ; \quad P_{r x}=\rho \nu\left(\frac{\partial V_{x}}{\partial r}+\frac{\partial V_{r}}{\partial x}\right) ; \quad P_{x x}=-p+2 \rho \nu \frac{\partial V_{x}}{\partial x} .
\end{gathered}
$$


If we take down the stress on the unperturbed surface of the shell, we can assume that $\bar{n}=\bar{n}_{r}$ and $\cos \left(\widehat{\bar{n}, \bar{n}_{r}}\right)=1, \cos (\widehat{\bar{n}, \bar{i}})=0$.

The equation of motion of a viscous incompressible fluid and the equation of continuity in a cylindrical coordinate system $(r, \Theta, x)$ in the case of axisymmetric flow are written in the form of $[6]$

$$
\begin{gathered}
\frac{\partial V_{r}}{\partial t}+V_{r} \frac{\partial V_{r}}{\partial r}+V_{x} \frac{\partial V_{r}}{\partial x}+\frac{1}{\rho} \frac{\partial p}{\partial r}=\nu\left(\frac{\partial^{2} V_{r}}{\partial r^{2}}+\frac{1}{r} \frac{\partial V_{r}}{\partial r}+\frac{\partial^{2} V_{r}}{\partial x^{2}}-\frac{V_{r}}{r^{2}}\right) \\
\frac{\partial V_{x}}{\partial t}+V_{r} \frac{\partial V_{x}}{\partial r}+V_{x} \frac{\partial V_{x}}{\partial x}+\frac{1}{\rho} \frac{\partial p}{\partial x}=\nu\left(\frac{\partial^{2} V_{x}}{\partial r^{2}}+\frac{1}{r} \frac{\partial V_{x}}{\partial r}+\frac{\partial^{2} V_{x}}{\partial x^{2}}\right), \\
\frac{\partial V_{r}}{\partial t}+\frac{V_{r}}{r}+\frac{\partial V_{x}}{\partial x}=0 .
\end{gathered}
$$

The fluid adhesion conditions in Lagrange's approach are satisfied at the boundary with the shell.

$$
\frac{\partial U}{\partial t}=V_{x}+U \frac{\partial V_{x}}{\partial x}-W \frac{\partial V_{x}}{\partial r} ; \quad-\frac{\partial W}{\partial t}=V_{r}+U \frac{\partial V_{r}}{\partial x}-W \frac{\partial V_{r}}{\partial r}
$$

We introduce dimensionless variables and parameters

$$
\begin{gathered}
V_{r}=w_{m} \frac{c_{0}}{l} v_{r} ; \quad V_{x}=w_{m} \frac{c_{0}}{R_{1}} v_{x} ; \quad r^{*}=\frac{r}{R_{1}} ; \quad t^{*}=\frac{c_{0}}{l} t ; \quad x^{*}=\frac{x}{l} ; \\
p=\frac{\rho \nu c_{0} l w_{m}}{R_{1}^{3}} P+p_{0} ; \quad \frac{R_{1}}{l}=\psi=O\left(\varepsilon^{\frac{1}{2}}\right) ; \quad \lambda=\frac{w_{m}}{R_{1}}=O(\varepsilon) ; \quad R e=\psi \frac{R_{1} c_{0}}{\nu} .
\end{gathered}
$$

By substituting (4.4) into Eq. (4.2) and the boundary condition (4.3), we obtain the equations and boundary conditions for the dimensionless components of the fluid velocity and pressure. By decomposing pressure and velocity components in powers of a small parameter $\lambda$

$$
P=P^{0}+\lambda P^{1}+\ldots, \quad v_{x}=v_{x}^{0}+\lambda v_{x}^{1}+\ldots, \quad v_{x}=v_{r}^{0}+\lambda v_{r}^{1}+\ldots,
$$

for the expansion first terms, we get the equations

$$
\begin{gathered}
\frac{\partial P^{0}}{\partial r^{*}}=0 ; \quad R e \frac{\partial v_{x}^{0}}{\partial t^{*}}+\frac{\partial P^{0}}{\partial x^{*}}=\frac{1}{r^{*}} \frac{\partial}{\partial r^{*}}\left(r^{*} \frac{\partial v_{x}^{0}}{\partial r^{*}}\right) ; \\
\frac{1}{r^{*}} \frac{\partial}{\partial r^{*}}\left(r^{*} v_{r}^{0}\right)+\frac{\partial v_{x}^{0}}{\partial x^{*}}=0
\end{gathered}
$$

and boundary conditions of the form

$$
\begin{gathered}
v_{r}^{0}=-\frac{\partial u_{3}}{\partial t^{*}} ; \quad v_{x}^{0}=\frac{u_{m} R_{1}}{w_{m} l} \frac{\partial u_{1}}{\partial t^{*}} \quad \text { if } \quad r^{*}=1 \\
r^{*} \frac{\partial v_{r}^{0}}{\partial r^{*}}=0 ; \quad r^{*} \frac{\partial v_{x}^{0}}{\partial r^{*}}=0 \quad \text { if } \quad r^{*}=0 .
\end{gathered}
$$

Now we define the stresses of the fluid on the shell in these variables. With an accuracy of order $\lambda, \psi$ we have

$$
q_{x}=-\left.\lambda \frac{\nu}{R_{1} c_{0}} \rho c_{0}^{2} \frac{\partial v_{x}}{\partial r^{*}}\right|_{r^{*}=1} ; \quad q_{n}=-p_{0}-\frac{\lambda}{\psi} \frac{\nu}{R_{1} c_{0}} \rho_{0} c_{0}^{2} P .
$$


By assuming the harmonic dependence of pressure, fluid velocity and elastic displacements of the shell on time, we find the exact solution of Eq. (4.6)

$$
\begin{aligned}
& P=\int\left\{2 \operatorname{Re}\left[(\alpha-1) \frac{1}{2} \frac{u_{m} \psi}{w_{m}} \frac{\partial^{2} u_{1}}{\partial \tau^{2}}-\alpha \int \frac{\partial^{2} u_{3}}{\partial \tau^{2}} d \xi\right]+\right. \\
&\left.+16 \gamma\left[\frac{1}{2} \frac{u_{m} \psi}{w_{m}} \frac{\partial^{2} u_{1}}{\partial \tau^{2}}-\int \frac{\partial u_{3}}{\partial \tau} d \xi\right]\right\} d \xi \\
&\left.\frac{\partial v_{x}}{\partial r^{*}}\right|_{r^{*}=1}=\operatorname{Re}(\alpha-1)\left(\frac{1}{2} \frac{u_{m} \psi}{w_{m}} \frac{\partial^{2} u_{1}}{\partial \tau^{2}}-\int \frac{\partial^{2} u_{3}}{\partial \tau^{2}} d \xi\right)+8 \gamma\left(\frac{1}{2} \frac{u_{m} \psi}{w_{m}} \frac{\partial u_{1}}{\partial \tau}-\int \frac{\partial^{2} u_{3}}{\partial \tau^{2}} d \xi\right),
\end{aligned}
$$

where

$$
\begin{gathered}
\alpha-1=\frac{2 \sqrt{R e} q_{0}-4 s_{0}}{\operatorname{Re} p_{0}-4 \sqrt{R e} q_{0}+4 s_{0}} ; \quad \alpha=\frac{\operatorname{Re} p_{0}-2 \sqrt{R e} q_{0}}{\operatorname{Re} p_{0}-4 \sqrt{R e} q_{0}+4 s_{0}}, \\
16 \gamma=\frac{4 \sqrt{R e} r_{0}}{\operatorname{Re} p_{0}-4 \sqrt{R e} q_{0}+4 s_{0}} .
\end{gathered}
$$

Here

$$
\begin{gathered}
p_{0}=b e r^{2} \sqrt{R e}+b e i^{2} \sqrt{R e}, \quad q_{0}=b e r \sqrt{R e}+b e i^{\prime} \sqrt{R e}-b e r^{\prime} \sqrt{R e} b e i \sqrt{R e} \\
r^{0}=b e r \sqrt{R e} b e i^{\prime} \sqrt{R e}+b e i \sqrt{R e} b e i^{\prime} \sqrt{R e}, \quad s_{0}=b e r^{\prime 2} \sqrt{R e}+b e i^{\prime 2} \sqrt{R e}
\end{gathered}
$$

ber and bei are zero-order Kelvin functions, and the prime denotes a derivative.

Note that

$$
\begin{gathered}
\alpha=\frac{4}{3}, \quad \gamma=1 \quad \text { when } R e \rightarrow 0, \\
\alpha=1, \quad \alpha-1=\sqrt{\frac{2}{R e}}, \quad \gamma=2 \frac{\sqrt{R e}}{8} \quad \text { when } R e>20 .
\end{gathered}
$$

Giving up the assumption of the flow parameters harmony in time in the problem under consideration, we apply the iteration method to the problem (4.6). At the first iteration step, we discard the local inertia term $(R e \ll 1)$ and obtain

$$
\begin{gathered}
P=16 \int\left(\frac{1}{2} \frac{u_{m} \psi}{w_{m}} \frac{\partial u_{1}}{\partial \tau}-\int \frac{\partial u_{3}}{\partial \tau} d \xi\right) d \xi \\
v_{x}=\frac{1}{4}\left(r^{* 2}-1\right) 16\left(\frac{1}{2} \frac{u_{m} \psi}{w_{m}} \frac{\partial u_{1}}{\partial \tau}-\int \frac{\partial u_{3}}{\partial \tau} d \xi\right)+\frac{u_{m} \psi}{w_{m}} \frac{\partial u_{1}}{\partial \tau} .
\end{gathered}
$$

In the second iteration step, by substituting the found solution into the local inertia term, we get

$$
\begin{gathered}
P=\int\left\{2 \operatorname{Re}\left[\frac{1}{6} \frac{u_{m} \psi}{w_{m}} \frac{\partial^{2} u_{1}}{\partial \tau^{2}}-\frac{4}{3} \int \frac{\partial^{2} u_{3}}{\partial \tau^{2}} d \xi\right]+16\left[\frac{1}{2} \frac{u_{m} \psi}{w_{m}} \frac{\partial^{2} u_{1}}{\partial \tau^{2}}-\int \frac{\partial u_{3}}{\partial \tau} d \xi\right]\right\} d \xi \\
\left.\frac{\partial v_{x}}{\partial r^{*}}\right|_{r^{*}=1}=\operatorname{Re} \frac{1}{3}\left(\frac{1}{2} \frac{u_{m} \psi}{w_{m}} \frac{\partial^{2} u_{1}}{\partial \tau^{2}}-\int \frac{\partial^{2} u_{3}}{\partial \tau^{2}} d \xi\right)+8\left(\frac{1}{2} \frac{u_{m} \psi}{w_{m}} \frac{\partial u_{1}}{\partial \tau}-\int \frac{\partial^{2} u_{3}}{\partial \tau^{2}} d \xi\right) .
\end{gathered}
$$

Formulas (4.11) coincide with the exact solution (4.9) for the harmonic laws of environmental parameters changes with $R e \ll 1$, which allows using these formulas for both nonharmonic parameters changes and for nonlinear equations of shell dynamics. 
It should be noted that the convergence of the iteration method was proved in [6]. Taking into account that variables (3.4) are introduced, and using the relation $c=\sqrt{1-\mu_{0}^{2}}$, we obtain, with an accuracy of order $\varepsilon$,

$$
\begin{gathered}
P=\sqrt{1-\mu_{0}^{2}}\left\{8\left(2 \int u_{30} d \xi-\frac{u_{m} R_{1}}{w_{m} l} u_{10}\right)-\right. \\
\left.-\frac{1}{3} \operatorname{Re}\left(8 u_{30}-\frac{u_{m} R_{1}}{w_{m} l} u_{10} \frac{\partial u_{10}}{\partial \xi}\right) \sqrt{1-\mu_{0}^{2}}\right\},
\end{gathered}
$$

where

$$
\begin{gathered}
\left.\frac{\partial v_{x}}{\partial r^{*}}\right|_{r^{*}=1}=\sqrt{1-\mu_{0}^{2}}\left\{4\left(u_{30}-\frac{u_{m} R_{1}}{w_{m} l} \frac{\partial u_{10}}{\partial \xi}\right)-\right. \\
\left.-\frac{1}{6} R e\left(2 \frac{\partial u_{30}}{\partial \xi}-\frac{u_{m} R_{1}}{w_{m} l} \frac{\partial^{2} u_{10}}{\partial \xi^{2}}\right) \sqrt{1-\mu_{0}^{2}}\right\} .
\end{gathered}
$$

As $\frac{u_{m} l}{w_{m} R_{1}} u_{30}=\mu_{0} \frac{\partial u_{10}}{\partial \xi}$, we get

$$
\begin{gathered}
q_{x}-\mu_{0} \frac{R}{l} \frac{\partial q_{n}}{\partial \xi}=\frac{\nu}{R_{1} c_{0}} \rho c_{0}^{2} 4 \sqrt{1-\mu_{0}^{2}} \frac{u_{m}}{l}\left[1-2 \mu_{0} \frac{R}{R_{1}}\right]^{2} \frac{\partial u_{10}}{\partial \xi}- \\
-\frac{R_{1}}{l} \rho c_{0}^{2} \frac{1}{6}\left(1-\mu_{0}^{2}\right) \frac{u_{m}}{l}\left[\left(1-2 \mu_{0} \frac{R}{R_{1}}\right)^{2}+3\left(2 \mu_{0} \frac{R}{R_{1}}\right)^{2} \frac{\partial^{2} u_{10}}{\partial \xi^{2}}\right] .
\end{gathered}
$$

Therefore, we have the equation

$$
\begin{gathered}
\frac{\partial^{2} u_{10}}{\partial \xi \partial \tau}+\frac{1}{\varepsilon} \frac{R^{2}}{l^{2}} \frac{\mu_{0}^{2} \sqrt{1-\mu_{0}^{2}}}{2} \frac{\partial^{4} u_{10}}{\partial \xi^{4}}- \\
-2 \frac{m}{E \varepsilon} \sqrt{1-\mu_{0}^{2}}\left(\mu_{1}+\mu_{2} \mu_{0}+\mu_{1} \mu_{0}^{2}\right)\left(\frac{u_{m}}{l}\right)^{2}\left(\frac{\partial u_{10}}{\partial \xi}\right)^{2} \frac{\partial^{2} u_{10}}{\partial \xi^{2}}+ \\
+k_{1} \frac{1}{\varepsilon} \frac{h_{0}}{R} \frac{\mu_{0}^{2}}{2 \sqrt{1-\mu_{0}^{2}}} \frac{\partial^{2} u_{10}}{\partial \xi^{2}}+\frac{1}{2} \frac{\varepsilon_{1}}{\varepsilon} \frac{\partial u_{10}}{\partial \xi}-\frac{1}{2} \frac{\varepsilon_{2}}{\varepsilon} \frac{\partial^{3} u_{10}}{\partial \xi^{3}} \mu_{0}^{2}= \\
=-\frac{1}{2} \frac{l}{\varepsilon \rho_{0} h_{0}}\left\{\frac{\nu}{R_{1} c_{0}} \rho 4\left[1-2 \mu_{0} \frac{R}{R_{1}}\right]^{2} \frac{\partial u_{10}}{\partial \xi}\right. \\
\left.-\frac{R_{1}}{l} \rho \frac{1}{6} \sqrt{1-\mu_{0}^{2}}\left[\left(1-2 \mu_{0} \frac{R}{R_{1}}\right)^{2}+3\left(2 \mu_{0} \frac{R}{R_{1}}\right)^{2}\right]^{2} \frac{\partial^{2} u_{10}}{\partial \xi^{2}}\right\} .
\end{gathered}
$$

The resulting equation generalizes the modified Korteweg-de Vries-Burgers equation for $\frac{\partial u_{10}}{\partial \xi}$.

With an accuracy of order $\varepsilon(4.15)$, we can put $R_{1}=R$.

Assuming $\frac{\partial u_{10}}{\partial \xi}=\phi, \eta=c_{1} \xi, t=c_{2} \tau$, we obtain the generalized modified Kortewegde Vries - Burgers (MKdV-B) equation

$$
\frac{\partial \phi}{\partial t}+\left(\sigma_{2}-\sigma_{5}-6 \phi^{2}\right) \frac{\partial \phi}{\partial \eta}+\frac{\partial^{3} \phi}{\partial \eta^{3}}-\sigma_{3} \frac{\partial^{2} \phi}{\partial \eta^{2}}+\left(\sigma_{4}+\sigma_{1}\right) \phi=0
$$


where

$$
\begin{gathered}
c_{1}=\left[\frac{2}{3} \frac{m}{E}\left(\frac{u_{m}}{l}\right)^{2} \frac{l^{2}}{R^{2}} \frac{\left(\mu_{1}+\mu_{2} \mu_{0}+\mu_{1} \mu_{0}^{2}\right)}{\mu_{0}^{2}}\right]^{\frac{1}{2}}, \\
c_{2}=c_{1} \frac{1}{3} \frac{m}{E \varepsilon} \sqrt{1-\mu_{0}^{2}}\left(\mu_{1}+\mu_{2} \mu_{0}+\mu_{1} \mu_{0}^{2}\right)\left(\frac{u_{m}}{l}\right)^{2},
\end{gathered}
$$

we assume that

$$
\begin{gathered}
\sigma_{1}=\frac{2}{c_{2}}\left(1-2 \mu_{0}\right)^{2} \frac{\rho l}{\varepsilon \rho_{0} h_{0}} \frac{\nu}{R_{1} c_{0}}, \quad \sigma_{2}=\frac{c_{1}}{c_{2}} \frac{\mu_{0}^{2}}{\sqrt{1-\mu_{0}^{2}}} \frac{k_{1}}{2} \frac{1}{\varepsilon} \frac{h_{0}}{R}, \\
\sigma_{3}=\frac{c_{1}^{2}}{c_{2}} \frac{1}{2} \frac{\varepsilon_{2}}{\varepsilon} \mu_{0}^{2}, \quad \sigma_{4}=\frac{1}{c_{2}} \frac{1}{2} \frac{\varepsilon_{1}}{\varepsilon} \\
\sigma_{5}=\frac{c_{1}}{c_{2}} \frac{1}{12} \sqrt{1-\mu_{0}^{2}} \frac{\rho l}{\rho_{0} h_{0} \varepsilon} \frac{R_{1}}{l}\left[\left(1-2 \mu_{0}\right)^{2}+3\left(2 \mu_{0}\right)^{2}\right] .
\end{gathered}
$$

Provided that $\left(\sigma_{4}+\sigma_{1}\right)=0$, in the absence of longitudinal structural damping $\left(\sigma_{4}=0\right)$ and $\mu_{0}=\frac{1}{2}$ for the incompressible material $\left(\sigma_{1}=0\right)$, the $\mathrm{MKdV}-\mathrm{B}$ equation

$$
\frac{\partial \phi}{\partial t}+\left(\sigma_{2}-\sigma_{5}-6 \phi^{2}\right) \frac{\partial \phi}{\partial \eta}+\frac{\partial^{3} \phi}{\partial \eta^{3}}-\sigma_{3} \frac{\partial^{2} \phi}{\partial \eta^{2}}=0
$$

has an exact solution in the form of a kink-antikink

$$
\phi= \pm \frac{1}{6} \sigma_{3} \pm k \tanh \left\{k\left[\eta+\left(2 k^{2}-\left(\sigma_{2}-\sigma_{5}\right)+\frac{1}{6} \sigma_{3}^{2}\right) t\right]\right\}
$$

The phase velocity is

$$
\frac{\omega}{k}=-2 k^{2}-\frac{1}{6} \sigma_{3}^{2}-\sigma_{5}+\sigma_{2}
$$

The wave velocity is

$$
C=\sqrt{\frac{E}{\rho_{0}}}\left(1-\varepsilon \frac{c_{2}\left(2 k^{2}+\frac{1}{6} \sigma_{3}^{2}+\sigma_{5}-\sigma_{2}\right)}{c_{1} \sqrt{1-\mu_{0}^{2}}}\right) .
$$

If the numerator of the fraction $2 k^{2}+\frac{1}{6} \sigma_{3}^{2}-\left(\sigma_{2}-\sigma_{5}\right)>0,\left(\left(\sigma_{2}-\sigma_{5}\right)<2 k^{2}+\frac{1}{6} \sigma_{3}^{2}\right)$, then the speed is subsonic. If the numerator of the fraction $2 k^{2}+\frac{1}{6} \sigma_{3}^{2}-\left(\sigma_{2}-\sigma_{5}\right)<0$, $\left(\left(\sigma_{2}-\sigma_{5}\right)>2 k^{2}+\frac{1}{6} \sigma_{3}^{2}\right)$, then the speed is supersonic. The effect of subgrade reaction $\sigma_{2}$ increases the wave velocity. At the same time, the fluid motion inertia of $\sigma_{5}$ reduces the wave speed. Thus, they act in different directions.

The constructing damping in the longitudinal direction $\sigma_{4}>0$ and the influence of a viscous fluid $\sigma_{1}>0$ affect the amplitude of the wave. Thus, they act in one direction. This effect is investigated by numerically solving the generalized modified Korteweg-de Vries-Burgers equation for $\left(\sigma_{4}+\sigma_{1}\right)>0$.

The wave number $k$ in (4.20) is arbitrary. 


\section{Computing experiment}

For a numerical study of the wave motion model of a physically nonlinear elastic shell with structural damping (energy dissipation) interacting with the surrounding elastic medium under the influence of the fluid, we write Eq. (4.16) in an integral form

$$
\oint_{\Omega} \phi d \eta-\left[\left(\sigma_{2}-\sigma_{5}\right) \phi-2 \phi^{3}-\sigma_{3} \frac{\partial \phi}{\partial \eta}+\frac{\partial^{2} \phi}{\partial \eta^{2}}\right] d t+\iint_{\Omega}\left(\sigma_{4}+\sigma_{1}\right) \phi d t d \eta=0
$$

for any area $\Omega$. To transfer a discrete formulation, let us compare $u_{j}^{n}=\phi\left(t_{n}, \eta_{j}\right)$ and select the basic contour shown in Figure 1.

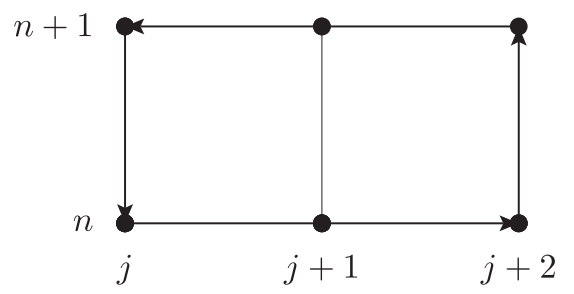

Fig. 1. Basic contour for equation (5.1)

Let us add the integral relations

$$
\begin{gathered}
\int_{m_{j}}^{m_{j+1}} u_{\eta} d \eta=u\left(t, \eta_{j+1}\right)-u\left(t, \eta_{j}\right), \\
\int_{m_{j}}^{m_{j+1}} u_{\eta \eta} d \eta=u_{\eta}\left(t, \eta_{j+1}\right)-u_{\eta}\left(t, \eta_{j}\right) .
\end{gathered}
$$

We use a trapezoid formula for integration in time and even derivatives in $\eta$ and an average value formula for odd derivatives in $\eta$. We assume $\left(t_{n+1}-t_{n}\right)=\tau,\left(\eta_{j+1}-t_{j}\right)=h$. We substitute all these expressions into formulas (5.1), (5.2) and, using the Gröbner basis method, we obtain the following difference scheme for Eq. (4.16) similar to the Crank-Nickolson scheme for the heat equation.

$$
\begin{gathered}
\frac{u_{j}^{n+1}-u_{j}^{n}}{\tau}+\left(\sigma_{2}-\sigma_{5}\right) \frac{\left(u_{j+1}^{n+1}-u_{j-1}^{n+1}\right)+\left(u_{j+1}^{n}-u_{j-1}^{n}\right)}{4 h}- \\
-2 \frac{\left(u_{j+1}^{3 n+1}-u_{j-1}^{3 n+1}\right)+\left(u_{j+1}^{3 n}-u_{j-1}^{3 n}\right)}{4 h}+ \\
+\frac{\left(u_{j+2}^{n+1}-2 u_{j+1}^{n+1}+2 u_{j-1}^{n+1}-u_{j-2}^{n+1}\right)+\left(u_{j+2}^{n}-2 u_{j+1}^{n}+2 u_{j-1}^{n}-u_{j-2}^{n}\right)}{4 h^{3}}- \\
-\sigma_{3} \frac{\left(u_{j+1}^{n+1}-2 u_{j}^{n+1}+u_{j-1}^{n+1}\right)+\left(u_{j+1}^{n}-2 u_{j}^{n}+u_{j-1}^{n}\right)}{4 h^{2}}+\left(\sigma_{4}+\sigma_{1}\right) \frac{u_{j}^{n+1}-u_{j}^{n}}{2}=0
\end{gathered}
$$

with the initial condition in the form of an exact solution (4.20) at $t=0$, choosing the plus sign, $k=\frac{1}{6}$. 


\section{The results and conclusions}

In the absence of environmental influences, structural damping in the longitudinal, normal directions and the influence of the fluid, the velocity and amplitude of the wave do not change. The movement occurs in the negative direction (Fig. 2). This means that the speed of movement is subsonic. The result of the computational experiment coincides with the exact solution, therefore, the difference scheme and the $\mathrm{KdV}-\mathrm{B}$ equation are adequate. The dimensionless deformation $\phi$, the dimensionless phase variable $\eta$, and dimensionless fast time $\tau$ are presented in Figs. 2-6.

In the absence of fluid influence, the influence of the surrounding elastic medium $\left(\sigma_{2}\right)$ leads to an increase in the wave velocity, up to a supersonic one. The presence of damping in the normal direction $\left(\sigma_{3}\right)$ changes the amplitude by constant volume (leads to the structure of the stretch shock wave $\phi>0$ ) and reduces the velocity of the wave. The presence of structural damping in the longitudinal direction $\left(\sigma_{4}\right)$ leads to a drop in the amplitude of the wave (Fig. 3).

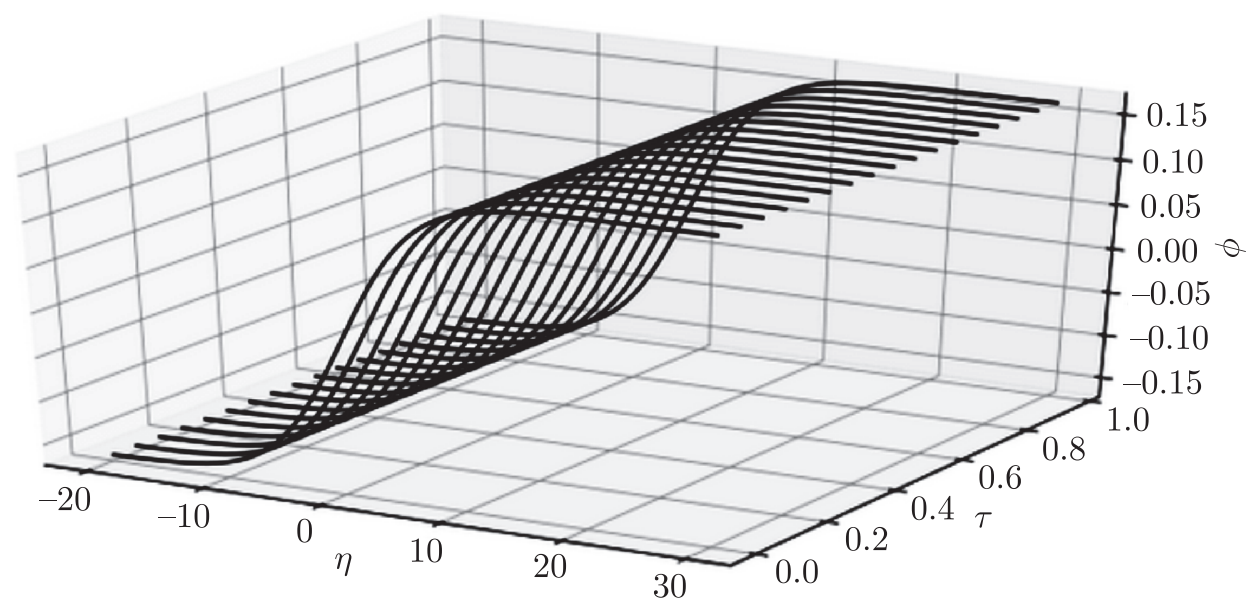

Fig. 2. The absence of environmental influence $\left(\sigma_{2}=0\right)$, structural damping in both the longitudinal $\left(\sigma_{4}=0\right)$ and normal $\left(\sigma_{3}=0\right)$ direction as well as the absence of fluid influence $\left(\sigma_{1}=0, \sigma_{5}=0\right)$.

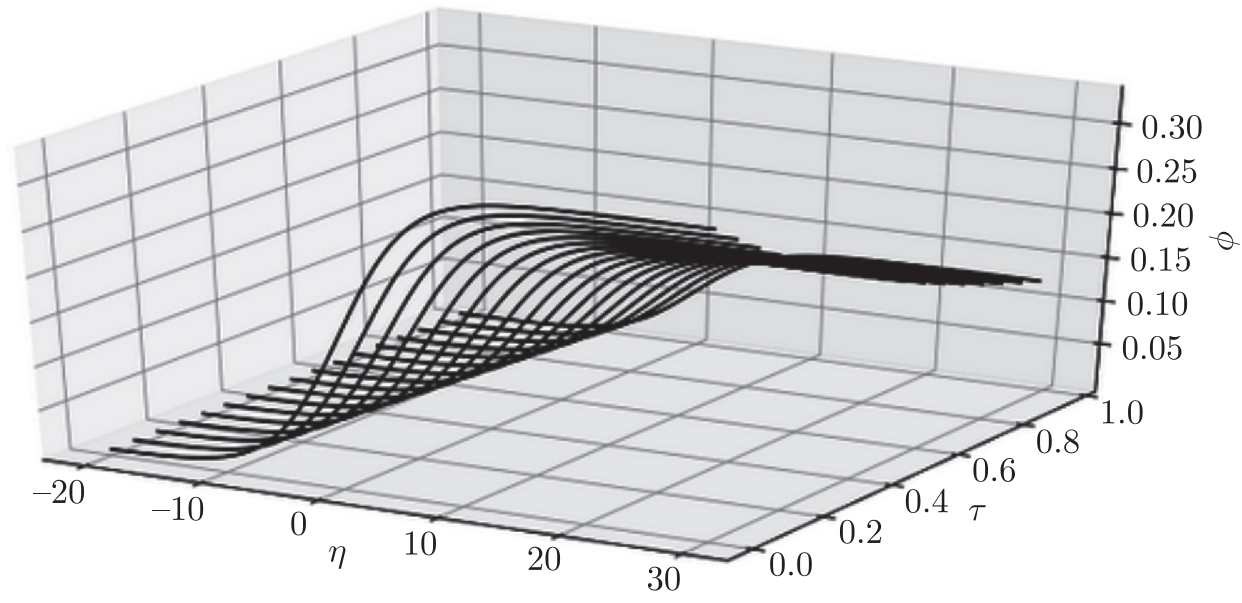

Fig. 3. The absence of fluid effect $\left(\sigma_{1}=0, \sigma_{5}=0\right) . \sigma_{2}=1, \sigma_{3}=1, \sigma_{4}=1$. 


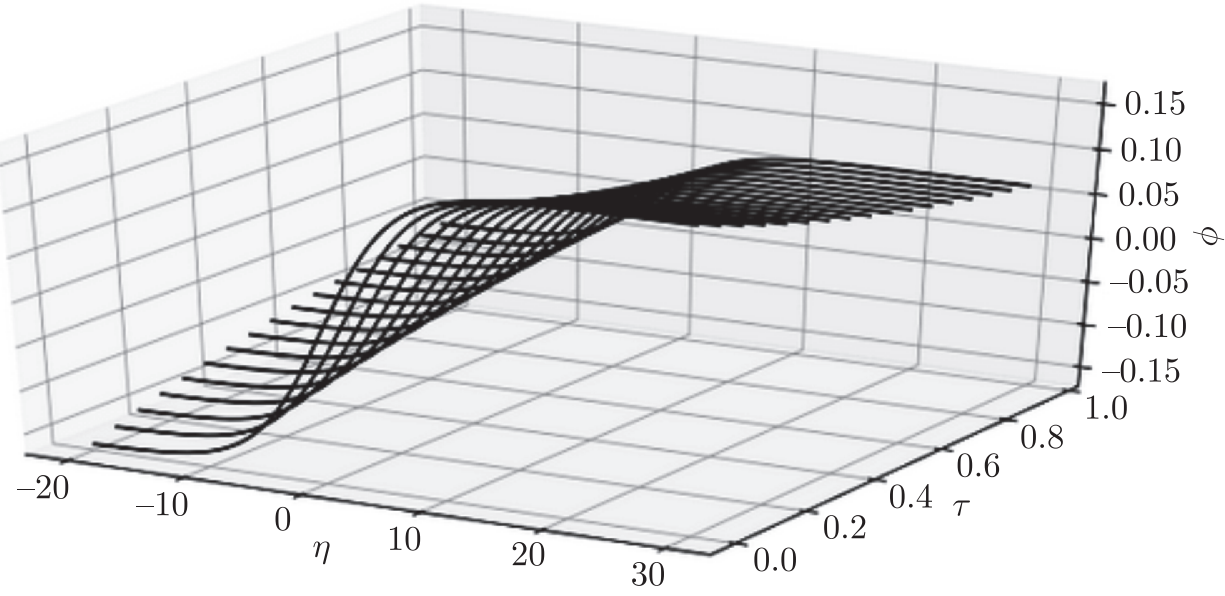

Fig. 4. The absence of environmental influence $\left(\sigma_{2}=0\right)$, structural damping both in the longitudinal $\left(\sigma_{4}=0\right)$ and normal $\left(\sigma_{3}=0\right)$ direction. $\sigma_{1}=1, \sigma_{5}=1$.

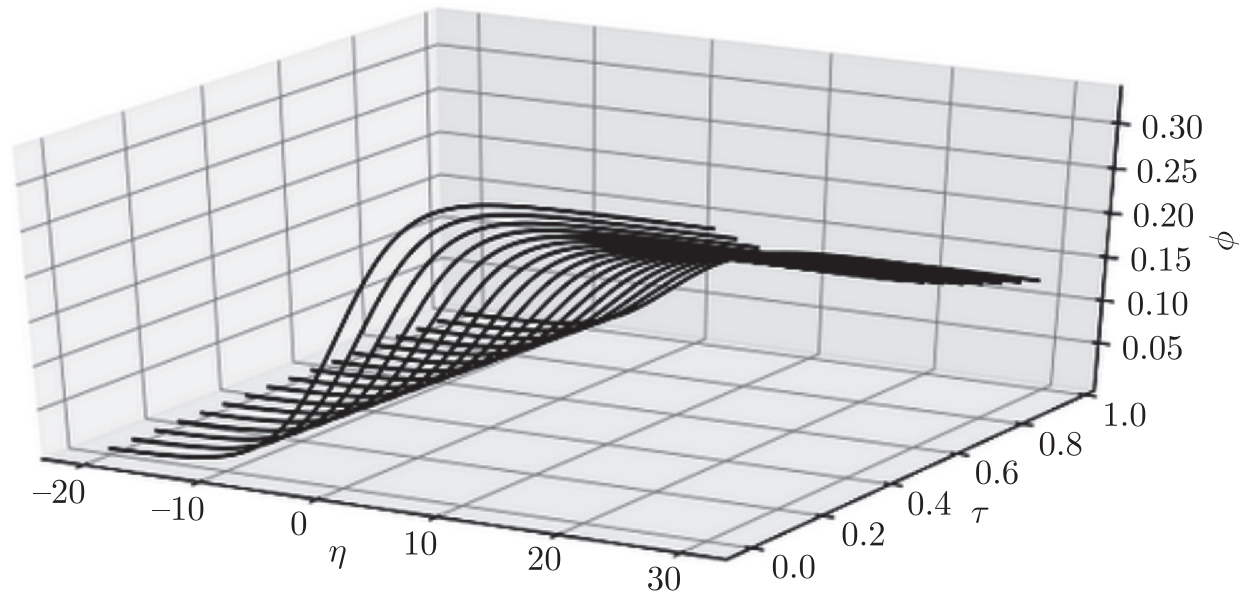

Fig. 5. All coefficients $\sigma_{1}, \sigma_{2}, \sigma_{3}, \sigma_{4}, \sigma_{5}$ are nonzero and equal to 1 , the stress from the fluid side is greater than the environmental influence $\left(\sigma_{2}<\sigma_{5}\right)$.

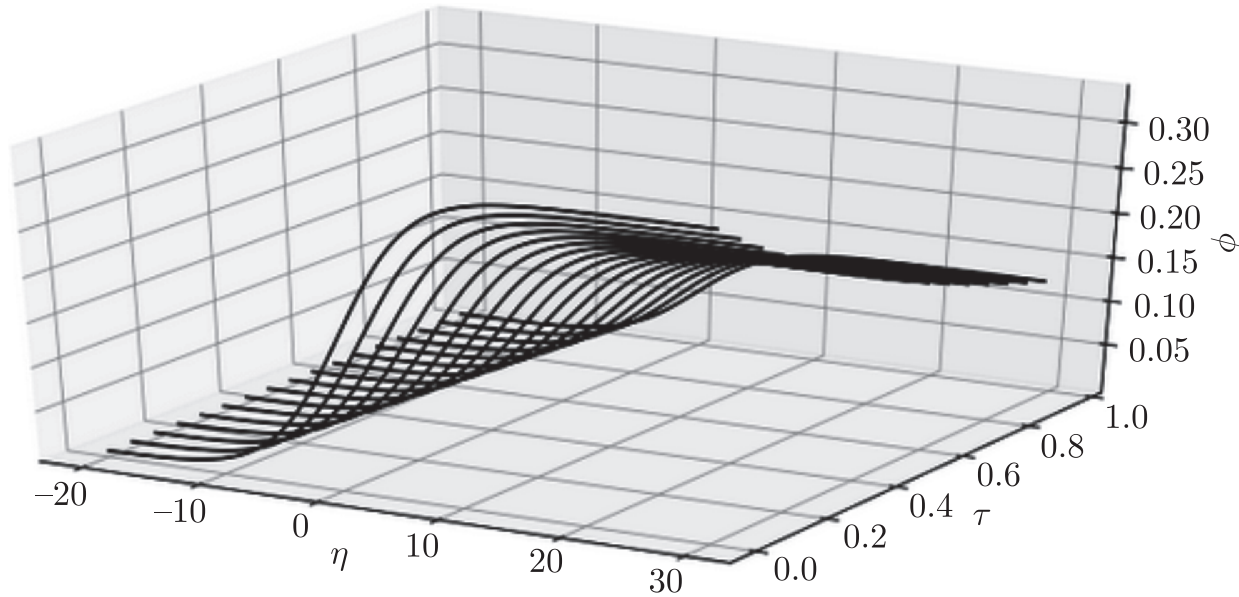

Fig. 6. All coefficients $\sigma_{1}, \sigma_{2}, \sigma_{3}, \sigma_{4}, \sigma_{5}$ are nonzero, the stress from the fluid side is less than the environmental influence $\left(\sigma_{2}>\sigma_{5}\right)$ 
The effect of viscous fluid stress on the shell $\left(\sigma_{1}\right)$ leads to a drop in the wave amplitude, and the inertia of fluid motion $\left(\sigma_{5}\right)$ leads to a decrease in the wave velocity (Fig. 4).

The presence of the medium $\left(\sigma_{2}\right)$ leads to an increase in the wave velocity, while the construction damping in the longitudinal direction $\left(\sigma_{4}\right)$ leads to a decrease in the wave amplitude. If the inertia of fluid motion $\left(\sigma_{5}\right)$ is greater than the influence of the medium $\left(\sigma_{2}<\sigma_{5}\right)$, the wave velocity decreases. The influence of fluid stress leads to a greater decrease in the wave amplitude (Fig. 5).

If the inertia of fluid motion $\left(\sigma_{5}\right)$ is less than the influence of the medium $\left(\sigma_{5}<\sigma_{2}\right)$, the increase in the wave velocity due to the presence of the medium $\left(\sigma_{2}\right)$ reduces (Fig. 6).

In the absence of the fluid influence, the influence of the elastic medium leads to an increase in the velocity of wave motion up to a supersonic one. The presence of construction damping in the normal direction changes the wave amplitude by constant volume (leads to the structure of the stretch shock wave) and reduces the velocity of wave motion. The presence of construction damping in the longitudinal direction reduces the wave amplitude.

The influence of the inertia of fluid movement reduces the deformation wave velocity, while fluid viscous stress on the shell reduces the wave amplitude.

The authors are grateful to the anonymous referees for a careful checking of the details and for helpful comments and suggestions that have improved this paper.

\section{References}

[1] Samarskii, A.A. and Mikhailov, A.P., Principles of Mathematical Modelling: Ideas, Methods, Examples, Numerical Insights, vol. 3, Boca Raton, Fla.: CRC, 2002.

[2] Ilyushin, A. A., Continuum Mechanics, 3rd ed., Moscow: MGU, 1990 (Russian).

[3] Kauderer, H., Nichtlineare Mechanik, Berlin: Springer, 1958.

[4] Volmir, A.S., Nonlinear Dynamics of Plates and Shells, Moscow: Nauka, 1972 (Russian).

[5] Zemlyanukhin, A. I. and Mogilevich, L. I., Nonlinear Waves in Cylindrical Shells: Solitons, Symmetry, Evolution, Saratov: SSU, 1999 (Russian).

[6] Loitsyanskiy, L. G., Mechanics of Liquids and Gases, 6th ed., New York: Begell House, 1995.

[7] Zemlyanukhin, A. I. and Mogilevich, L. I., Nonlinear Deformation Waves in Cylindrical Shells, Izv. Vyssh. Uchebn. Zaved. Prikl. Nelin. Dinam., 1995, vol. 3, no. 1, pp. 52-58 (Russian).

[8] Erofeev, V.I. and Klyueva, N.V., Solitons and Nonlinear Periodic Strain Waves in Rods, Plates, and Shells (A Review), Acoust. Phys., 2002, vol.48, no.6, pp.643-655; see also: Akust. Zh., 2002, vol. 48, no. 6, pp. $725-740$.

[9] Bochkarev, A. V., Zemlyanukhin, A. I., and Mogilevich, L.I., Solitary Waves in an Inhomogeneous Cylindrical Shell Interacting with an Elastic Medium, Acoust. Phys., 2017, vol. 63, no. 2, pp. 145-151; see also: Akust. Zh., 2017, vol. 63, no. 2, pp. 145-151.

[10] Krysko, V.A., Zhigalov, M.V., and Saltykova, O.A., Nonlinear Dynamics of Beams of EulerBernoulli and Timoshenko Type, Izv. Vyssh. Uchebn. Zaved. Mashinostr., 2008, no.6, pp.7-27 (Russian).

[11] Zemlyanukhin, A. I., Bochkarev, A. V., and Mogilevich, L. I., Solitary Longitudinal-Bending Waves in Cylindrical Shell Interacting with a Nonlinear Elastic Medium, Herald of the Bauman Moscow State Technical University. Series Natural Sciences, 2018, no.1(76), pp. 47-60 (Russian).

[12] Ageev, R. V., Kuznetsova, E. L., Kulikov, N.I., Mogilevich, L.I., and Popov, V.S., Mathematical Model of Movement of a Pulsing Layer of Viscous Liquid in the Channel with an Elastic Wall, PNRPU Mech. Bull., 2014, no. 3, pp.17-35 (Russian). 
[13] Lekomtsev, S. V., Finite-Element Algorithms for Calculation of Natural Vibrations of ThreeDimensional Shells, Comput. Contin. Mech., 2012, vol. 5, no. 2, pp. 233-243 (Russian).

[14] Bochkarev, S. A. and Matveenko, V.P., Stability of Coaxial Cylindrical Shells Containing Rotating Fluid Flow, Comput. Contin. Mech., 2013, vol. 6, no. 1, pp. 94-102 (Russian).

[15] Mamaev, I. S., Tenenev, V. A., and Vetchanin, E. V., Dynamics of a Body with a Sharp Edge in a Viscous Fluid, Rus. J. Nonlin. Dyn., 2018, vol. 14, no. 4, pp. 473-494.

[16] Borisov, A. V., Mamaev, I. S., and Vetchanin, E. V., Self-Propulsion of a Smooth Body in a Viscous Fluid under Periodic Oscillations of a Rotor and Circulation, Regul. Chaotic Dyn., 2018, vol. 23, nos. $7-8$, pp. 850-874.

[17] Kuzenov, V.V. and Ryzhkov, S. V., Approximate Method for Calculating Convective Heat Flux on the Surface of Bodies of Simple Geometric Shapes, J. Phys. Conf. Ser., 2017, vol. 815, 012024, 8 pp.

[18] Ryzhkov, S. V. and Kuzenov, V. V., Analysis of the Ideal Gas Flow over Body of Basic Geometrical Shape, Int. J. Heat Mass Transf., 2019, vol. 132, pp. 587-592.

[19] Vetchanin, E. V., Mamaev, I. S., and Tenenev, V.A., The Self-Propulsion of a Body with Moving Internal Masses in a Viscous Fluid, Regul. Chaotic Dyn., 2013, vol.18, nos.1-2, pp. 100-117.

[20] Andrejchenko, K. P. and Mogilevich, L. I., On the Dynamics of Interaction between a Compressible Layer of a Viscous Incompressible Fluid and Elastic Walls, Izv. Akad. Nauk. Mekh. Tverd. Tela, 1982, no. 2, pp. 162-172 (Russian).

[21] Gerdt, V.P., Blinkov, Yu. A., and Mozzhilkin, V.V., Gröbner Bases and Generation of Difference Schemes for Partial Differential Equations, SIGMA Symmetry Integrability Geom. Methods Appl., 2006, vol. 2, Paper 051, 26 pp.

[22] Ovcharov, A. A. and Brylev, I. S., Mathematical Model of Deformation of Nonlinear Elastic Reinforced Conical Shells under Dynamic Loading, Sovremennye Problemy Nauki i Obrazovaniya, 2014, no. 3, 8 pp. (Russian).

[23] Fel'dshtejn, V. A., Elastic Plastic Deformations of a Cylindrical Shell with a Longitudinal Impact, in Waves in Inelastic Media, Kishinev: Akad. Nauk MSSR, 1970, pp. 199-204 (Russian). 